\title{
Modelización del Efecto del Compromiso Escolar sobre el Rendimiento Escolar en Cinco Países de Iberoamérica
}

\author{
Modeling the School Engagement Effect on School Performance in Five \\ Iberoamerican Countries
}

\author{
Edgardo Miranda-Zapata ${ }^{1}$, Laura Lara $^{2}$ y Mahia Saracostti ${ }^{3}$
}

\begin{abstract}
Resumen
En el presente estudio se buscó establecer el ajuste de un modelo de mediación del Compromiso escolar entre la relación de factores contextuales y el rendimiento escolar, en estudiantes de Chile, Colombia, España, Perú y Uruguay. El modelo vincula causalmente el compromiso escolar (afectivo, cognitivo y conductual) con la asistencia a clases y el rendimiento escolar; incluyendo además el efecto indirecto de factores contextuales (familia, profesorado y pares). Se evaluaron estudiantes de enseñanza primaria y secundaria, con una edad promedio de 14.73 años $(D S=1.59)$. Los tamaños muestrales fluctuaron entre 343 y 438 participantes por país. El modelo presenta un ajuste aceptable en los cinco países (RMSEA=.043-.080; $C F I=.910-.961 ; T L I=.897-.958)$, con divergencias en las dimensiones del compromiso escolar que presentan efectos significativos sobre las variables dependientes.
\end{abstract}

Palabras clave: asistencia a clases, compromiso escolar, factores contextuales, modelo teórico, rendimiento escolar

\begin{abstract}
The objective of this study to understand school engagement as mediator variable between contextual factors and school achievement in students from Chile, Colombia, Spain, Perú and Uruguay. The causal model links school engagement (affective, cognitive, and behavioral) with class attendance and school performance; while also considering the indirect effect of contextual factors (family, teachers and peers). Students from primary and secondary education were evaluated, with a mean age of 14.73 years-old $(\mathrm{SD}=1.59)$. The sample sizes ranged between 343 and 438 participants per country. The model presented an acceptable adjustment in the five countries (RMSEA=.043-.080; CFI=.910-.961; TLI=.897-.958), with exception of the school engagement dimensions that presented significant effects on the dependent variables.
\end{abstract}

Keywords: class attendance, school engagement, contextual factors, theoretical model school achievement

\footnotetext{
${ }^{1}$ Edgardo Miranda-Zapata. Doctor en Metodología de las Ciencias del Comportamiento y de la Salud. Académico e Investigador. Núcleo Científico Tecnológico en Ciencias Sociales y Humanidades, Universidad de La Frontera. Universidad de la Frontera, Avenida Francisco Salazar, 01145. Temuco, Chile. Tel.: +56 452596732. Correo: edgardo.miranda@ufrontera.cl

${ }^{2}$ Doctora en Psicología. Profesora Titular. Universidad Autónoma de Chile, 5 poniente 1670, Talca, Chile. Tel.: +56 712735770. Correo: 1larav@uautonoma.cl

${ }^{3}$ Doctora en Social Welfare. Profesora Titular de la Escuela de Trabajo Social de la Facultad de Ciencias Sociales. Directora de la Cátedra UNESCO Niñez, Juventud, Educación y Sociedad. Universidad de Valparaíso. Universidad de Valparaíso, Blanco 951, Valparaíso, Chile. Tel.: +56 998293161. Correo: mahia.saracostti@uv.cl
} 


\section{Introducción}

El presente estudio considera el compromiso escolar (CE) desde una perspectiva estructural de tres dimensiones: afectiva, cognitiva y conductual (Appleton et al., 2008; Fredricks, Blumenfeld, \& Paris, 2004; Fredricks, Filsecker, \& Lawson, 2016). En ella, el compromiso afectivo se entiende como la respuesta emocional del/la estudiante hacia el establecimiento educativo y su proceso de aprendizaje, incluyendo un sentimiento de involucramiento con el colegio. El compromiso cognitivo se vincula con la consciencia y la voluntad de realizar el esfuerzo de comprender ideas y desarrollar habilidades. Refleja la disposición del/la estudiante para utilizar sus habilidades cognitivas en el aprendizaje. Finalmente, el compromiso conductual se refiere a la participación del/la estudiante en el ámbito académico y actividades extracurriculares. Incluye interacciones y respuestas dentro de la sala de clases, dentro del colegio y también fuera de él.

El CE es una variable relevante para la permanencia o deserción del/la estudiante en el sistema escolar (Kiefer, Alley, \& Ellerbrock, 2015; Melo, Failache, \& Machado, 2015; Sahin, Arseven, \& Kiliç, 2016). Sobre él influyen factores de contexto (FC) como la familia, pares y el profesorado (Ansong et al., 2017; FernándezZabala, Goñi, \& Zulaika, 2015; Yusof, Ang, \& Oei, 2017).

El estudio de Roundfield, Sánchez y McMahon (2016) evidencia que los FC escolares $\mathrm{y}$, en particular, los grupos de pares inciden en las tres dimensiones del $\mathrm{CE}$, mientras que la familia afectaría particularmente el compromiso conductual y cognitivo de los/las adolescentes. Por su parte Quin, Heerde y Toumbourou (2018) destacan que los factores contextuales/relacionales influyen fuertemente en el CE de los/las estudiantes, de manera que los factores asociados a la familia y los/las compañeros(as) de escuela realizan contribuciones significativas a algunos indicadores académicos y emocionales del CE, así como con algunos problemas socio-educacionales más amplios como el ausentismo o el abandono escolar.

Actualmente, el CE está siendo ampliamente estudiado en su relación, e influencia sobre, el rendimiento académico (Froiland \& Worrell,
2016; Gutiérrez, Sancho, Galiana, \& Tomás, 2018; Korobova \& Starobin, 2015; Li, Chen, \& Li, 2020; Miranda-Zapata et al., 2018; Salanova, Schaufeli, Martínez, \& Bresó, 2010; Tomás, Gutiérrez, \& Fernández, 2016; Veiga et al., 2016). Así, se ha encontrado que el CE es un predictor estadísticamente significativo del desempeño académico que alcanzan los/as estudiantes (Appleton, Christenson, \& Furlong, 2008; Archambault, Janosz, Fallu, \& Pagani, 2009; Weiss, Carolan, \& Baker-Smith, 2010).

El estudio de González, Paoloni, Donolo y Rinaudo (2015) analiza las relaciones estructurales entre las variables facilitadores de la motivación escolar y el rendimiento académico, así como el papel del CE y la desafección como mediadores de los efectos del control y el valor sobre el rendimiento en estudiantes españoles. Sus resultados confirmaron las hipótesis: el control y el valor predijeron significativamente el compromiso, la desafección y el rendimiento; el compromiso y la desafección predijeron, a su vez, el rendimiento y mediaron parcialmente los efectos del control y el valor sobre el rendimiento.

Con base en lo expuesto hasta el momento se puede considerar que es posible intervenir los factores del contexto en el cual se desarrollan los/as estudiantes, los cuales presentarán un efecto sobre su $\mathrm{CE}$, haciendo de él un concepto moldeable o adaptable (Dotterer, McHale, \& Crouter, 2007; Lam, Wong, Yang, \& Liu, 2012; Reschly \& Christenson, 2012), que tendría un efecto sobre el rendimiento escolar y la permanencia en el sistema escolar. Lo que constituye un aspecto relevante dado que la deserción escolar y repetición de curso son consideradas como problemas en países de Iberoamérica como Chile (Espinoza, González, McGinn, \& Castillo, 2020), Colombia (Flotts et al., 2016), España (Martínez-Novillo, 2017), Perú (MINEDU, 2007) y Uruguay (Acevedo, 2020). Así, el presente estudio se propone determinar el ajuste de un modelo que vincula indirectamente los factores contextuales (familia, pares y profesorado) con el rendimiento escolar (porcentaje de logro (basado de en el promedio de notas) y la asistencia a clases (porcentaje de asistencia)), a través del compromiso escolar (afectivo, cognitivo y conductual) (Figura 1), en cinco países de Iberoamérica (Chile, Colombia, 


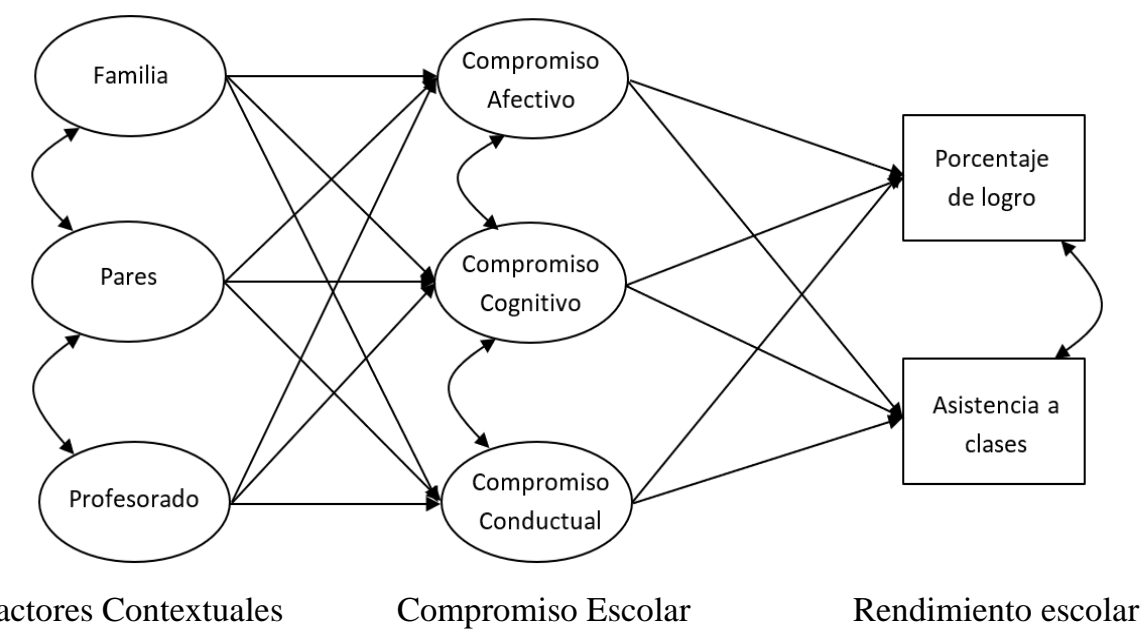

Figura 1. Diagrama de rutas del Modelo teórico de mediación del Compromiso Escolar en la relación entre Factores Contextuales y Rendimiento Escolar*

(*) Por simplicidad se omiten los modelos de medida y los errores de predicción.

España, Perú y Uruguay). En relación con las hipótesis $(\mathrm{H})$ del estudio, esperamos que el modelo presente un ajuste aceptable en todos los países participantes (H1). Así mismo, esperamos que los parámetros estructurales presenten variaciones de significancia estadística entre los países (H2).

\section{Método}

Se utilizó un diseño descriptivo correlacional transversal, basado en modelos causales para valorar el ajuste de un modelo teórico de mediación del compromiso escolar en la relación de los factores contextuales y el rendimiento escolar (porcentaje de logro y asistencia a clases).

\section{Muestra}

Se utilizó un muestreo no probabilístico por conveniencia, accediendo a un total de 1926 estudiantes de los cinco países (409 de Chile, 438 de Colombia, 343 de España, 355 de Perú y 381 de Uruguay). Los participantes presentaron una edad promedio de 14.73 años $(D S=1.59)$ y se encontraban cursando los niveles de $7^{\circ}$ Básico a $3^{\circ}$ Medio.

\section{Instrumentos}

- Cuestionario de Compromiso Escolar. Desarrollado por Lara et al. (2018). Es un cuestionario que mide las tres dimensiones del compromiso escolar: afectivo (10 ítems), conductual ( 7 ítems) y cognitivo (12 ítems). Los 29 ítems se encuentran en escala tipo Likert de 5 puntos (desde 1: nunca o casi nunca hasta 5: siempre o casi siempre). El instrumento presenta evidencia a favor de la validez estructural en los 5 países (Tabla 1), con valores de RMSEA entre .035 y .068 , CFI entre .982 у .988 у TLI entre .944 y .983 y buenos índices de fiabilidad como consistencia interna (Tabla 2) con valores de omega entre .68 y .91 para el compromiso afectivo, entre .84 y .98 para el compromiso conductual y entre .81 y .91 para el compromiso cognitivo. Cabe señalar que la estructura del cuestionario fue distinta entre los países (ver artículo de validación en esta misma edición).

Tabla 1. Índices de bondad de ajuste del modelo de medida de Compromiso Escolar

\begin{tabular}{lccc}
\hline & RMSEA & CFI & TLI \\
\hline Chile & .068 & .984 & .983 \\
Colombia & .052 & .983 & .975 \\
Uruguay & .043 & .986 & .978 \\
España & .058 & .982 & .973 \\
Perú & .035 & .988 & .944 \\
\hline
\end{tabular}

Tabla 2. Coeficiente Omega para las dimensiones del Cuestionario de Compromiso Escolar

\begin{tabular}{lccccc}
\hline & Chile & Colombia & España & Perú & Uruguay \\
\hline Afectivo & .91 & .81 & .81 & .81 & .68 \\
Conductual & .98 & .89 & .91 & .84 & .85 \\
Cognitivo & .91 & .81 & .83 & .90 & .89 \\
\hline
\end{tabular}

- Cuestionario de Factores Contextuales. Desarrollado por Universidad de la Frontera y la Universidad Autónoma (2016). Es un cuestionario 
que valora 3 dimensiones del contexto de los/as estudiantes: familia (3 ítems), profesorado (8 ítems) y pares ( 7 ítems). Los 18 ítems se encuentran en escala tipo Likert de 5 puntos (desde 1: nunca o casi nunca hasta 5: siempre o casi siempre). El instrumento presenta evidencia a favor de la validez estructural en los 5 países (Tabla 3), con valores de RMSEA=.053-.098, $C F I=.933-.993$ y $T L I=.915-.984$ y buenos índices de fiabilidad como consistencia interna (Tabla 4) con valores de omega entre .73 y .79 para el factor contextual familia, entre .86 y .90 para el factor contextual profesorado y entre .77 y .90 para el factor contextual pares. Cabe señalar que la estructura del cuestionario fue distinta entre los países (ver artículo de validación en esta misma edición).

Tabla 3. Índices de bondad de ajuste del modelo de medida de Factores Contextuales

\begin{tabular}{lccc}
\hline & RMSEA & CFI & TLI \\
\hline Chile & .088 & .933 & 9.23 \\
Colombia & .093 & .949 & .915 \\
Uruguay & .053 & .993 & .984 \\
España & .098 & .962 & .937 \\
Perú & .082 & .967 & .949 \\
\hline
\end{tabular}

Tabla 4. Coeficiente Omega para las dimensiones del Cuestionario de Factores Contextuales

\begin{tabular}{lccccc}
\hline & Chile & Colombia & España & Perú & Uruguay \\
\hline Familia & .79 & .73 & .79 & .77 & .74 \\
Profesorado & .89 & .86 & .90 & .90 & .87 \\
Pares & .88 & .77 & .90 & .89 & .87 \\
\hline
\end{tabular}

\section{Procedimiento}

El/la adulto(a) responsable de cada estudiante firmó un consentimiento informado en el que se explicaban las condiciones de confidencialidad y aplicación de los instrumentos de evaluación. Cada estudiante firmó un asentimiento estableciendo su voluntad de participar en la investigación. El procedimiento de este estudio fue revisado y aprobado por el Comité de Ética Científica de la Universidad de La Frontera, Temuco, Chile.

Los instrumentos validados para valorar el nivel de compromiso escolar y los factores contextuales de los/as estudiantes, fueron aplicados en los establecimientos educativos, a través de la plataforma online de Evaluación del Compromiso Escolar (www.compromisoescolar.cl). El sistema de la plataforma favoreció que no existan datos perdidos, ya que el/la participante no podía avanzar entre páginas de preguntas ni finalizar la encuesta si existían ítems sin responder.

Se solicitó a los/as directores/as de los establecimientos educativos acceso a la información relativa al rendimiento de los/as estudiantes, específicamente sobre el promedio general de Notas, y el porcentaje de Asistencia de cada estudiante. Las notas fueron transformadas a porcentaje de logro, de acuerdo con la escala de notas de cada país, utilizando la siguiente fórmula: $(\mathrm{x}-\mathrm{min} / \max -\min ) * 100$. Donde $\mathrm{x}$ es la nota del estudiante, min es la nota mínima de la escala de notas del país y max es la nota máxima de la escala de notas del país.

Para la realización de análisis descriptivos se utilizó el programa estadístico IBM SPSS v. 23. El programa Mplus v. 7.3 fue utilizado para la realización de los análisis de modelos de ecuaciones estructurales. Se trabajó sobre la matriz de correlaciones policóricas utilizando el estimador Cuadrados Mínimos Ponderados Robusto (WLSMV), acorde con lo planteado por Li (2014), considerando la estructura avalada por los datos de cada país (ver artículos de validación en esta misma edición).

Para valorar el ajuste del modelo a los datos se utilizaron los índices de bondad de ajuste habitualmente informados para análisis de estructuras de covarianzas, tales como el índice de bondad de ajuste Raíz Cuadrada Media del Error de Aproximación (Root Mean Square Error of Approximation; RMSEA), para el cual valores inferiores a .06 indican un buen ajuste y valores inferiores a .08 indican un ajuste aceptable (Marsh, Hau, \& Grayson, 2005). Los índices de Tuker-Lewis (TLI o Non-Normed Fit Index (NNFI)) y de Ajuste Comparativo (Comparative Fit Index; CFI), para los cuales valores sobre .90 para indicar un ajuste aceptable y valores sobre .95 indican un buen ajuste (Kline, 2010).

Tabla 5. Índices de bondad de ajuste del Modelo de ecuaciones estructurales

\begin{tabular}{lccc}
\hline País & RMSEA & CFI & TLI \\
\hline Chile & .065 & .954 & .951 \\
Colombia & .067 & .925 & .918 \\
España & .070 & .926 & .919 \\
Perú & .043 & .961 & .958 \\
Uruguay & .080 & .910 & .897 \\
\hline
\end{tabular}




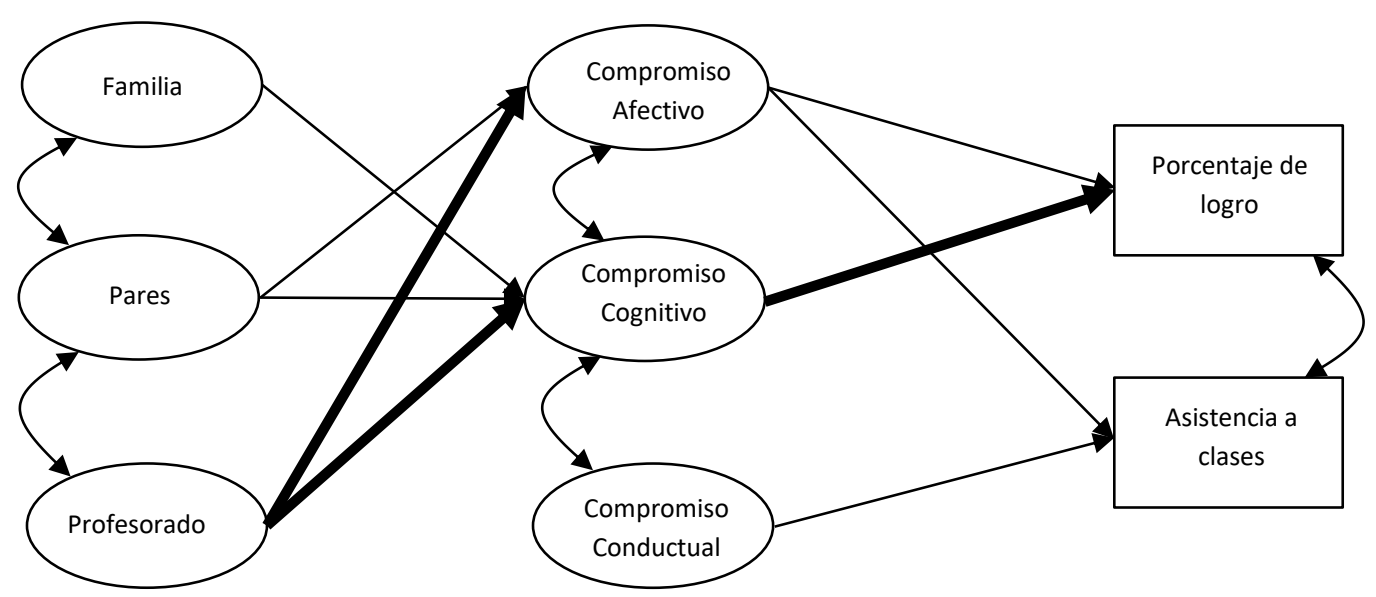

Factores Contextuales

Compromiso Escolar

Rendimiento escolar

Figura 2. Diagrama de rutas estadísticamente significativas del Modelo teórico de mediación del Compromiso

Escolar en la relación entre Factores Contextuales y Rendimiento Escolar, en participantes de Chile

Efecto de magnitud alta $(\geq .50)$

- Efecto de magnitud media $(>.30 \mathrm{y}<.50)$

Efecto de magnitud baja $(\leq .30)$

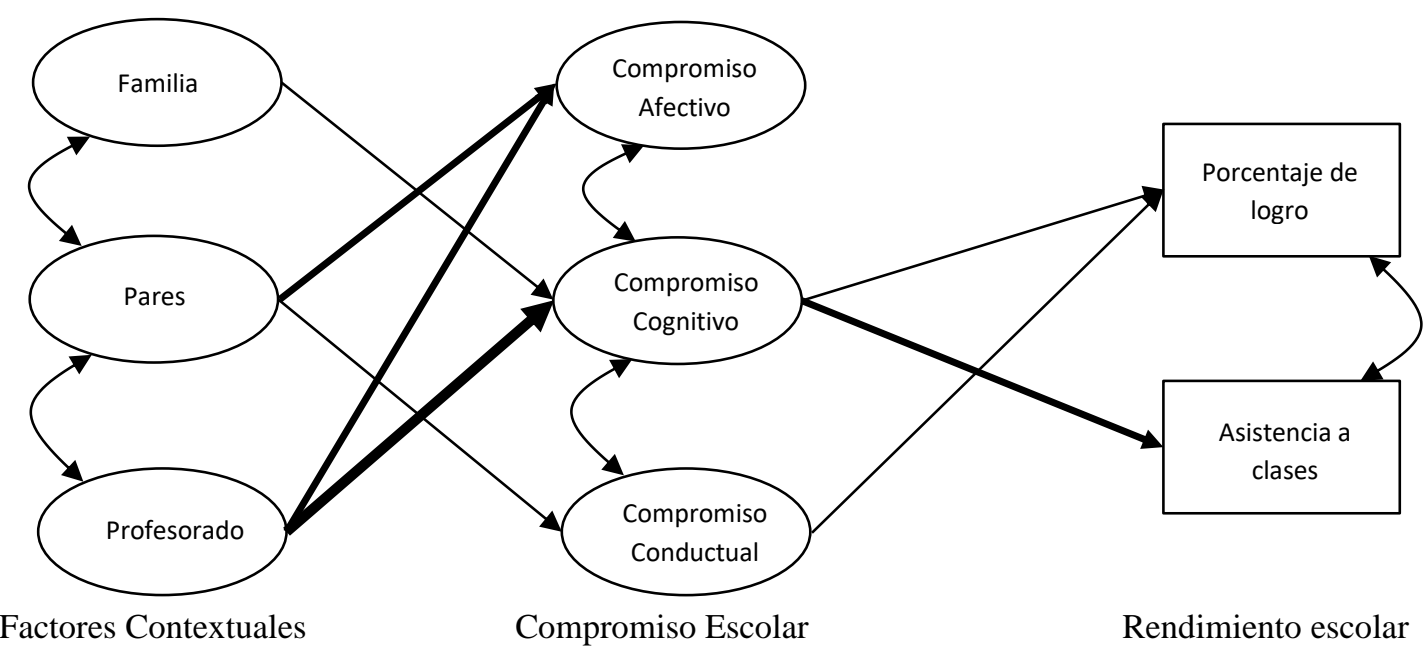

Figura 3. Diagrama de rutas estadísticamente significativas del Modelo teórico de mediación del Compromiso

Escolar en la relación entre Factores Contextuales y Rendimiento Escolar, en participantes de Colombia

Efecto de magnitud alta $(\geq .50)$

Efecto de magnitud media $(>.30 \mathrm{y}<.50)$

Efecto de magnitud baja $(\leq .30)$

\section{Resultados}

El modelo presenta un ajuste aceptable en los cinco países (Tabla 5), lo que satisface la H1.

Existe variabilidad en cuanto a los parámetros estructurales que resultaron estadísticamente significativos en los distintos países lo que da sustento a la H2. A continuación, se presentan los diagramas de ruta del modelo de cada país, especificando los parámetros estructurales estadísticamente significativos. Por simplicidad, se omitieron los ítems y sus saturaciones.

En la muestra de participantes de Chile, el modelo (Figura 2) presentó tres efectos de magnitud alta, el efecto del factor contextual profesorado sobre el compromiso escolar afectivo $(\gamma=.669, p<.001)$, el efecto del factor contextual profesorado sobre el compromiso escolar cognitivo $(\gamma=.645, \quad p<.001)$ y el efecto del compromiso escolar cognitivo sobre el porcentaje de logro $(\beta=.523, p<.001)$. Además, de un efecto de magnitud media entre el factor contextual profesorado y el compromiso escolar conductual $(\gamma=.459, p<.001)$.

En la muestra de participantes de Colombia, el modelo (Figura 3) presentó un efecto de magnitud alta, el efecto del factor contextual profesorado 


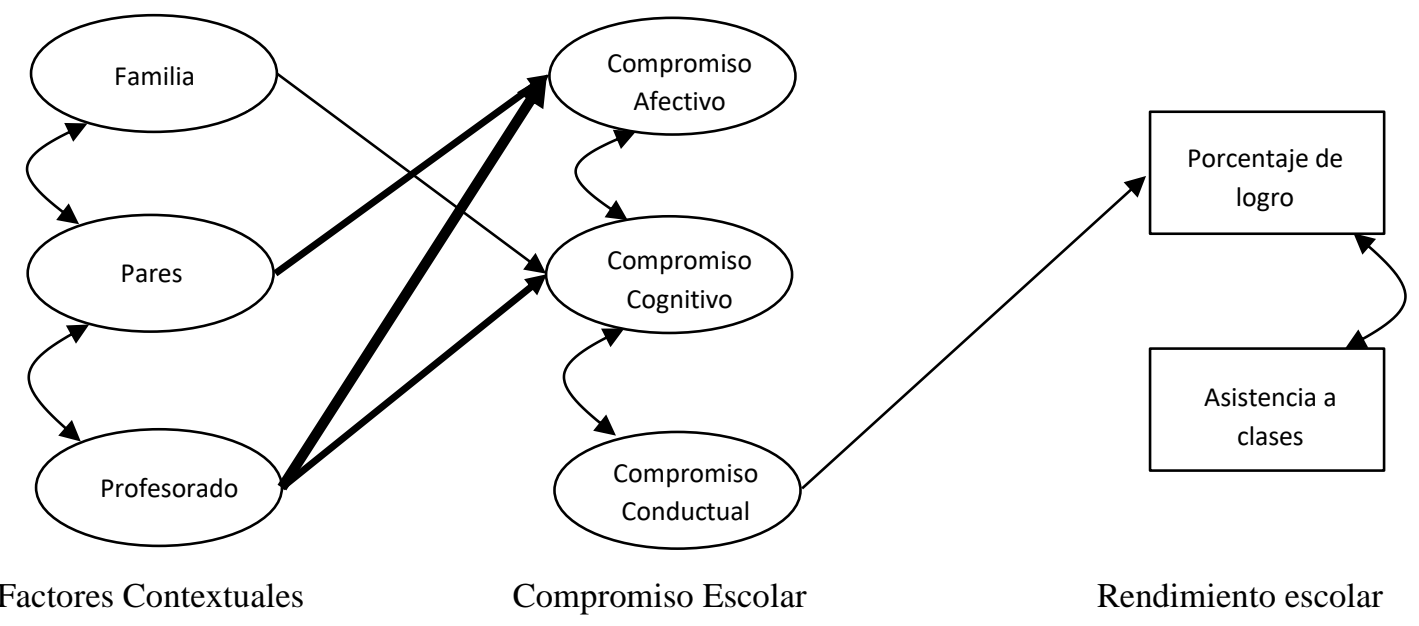

Figura 4. Diagrama de rutas estadísticamente significativas del Modelo teórico de mediación del Compromiso

Escolar en la relación entre Factores Contextuales y Rendimiento Escolar, en participantes de España

Efecto de magnitud alta $(\geq .50)$

Efecto de magnitud media $(>.30 \mathrm{y}<.50)$

Efecto de magnitud baja $(\leq .30)$

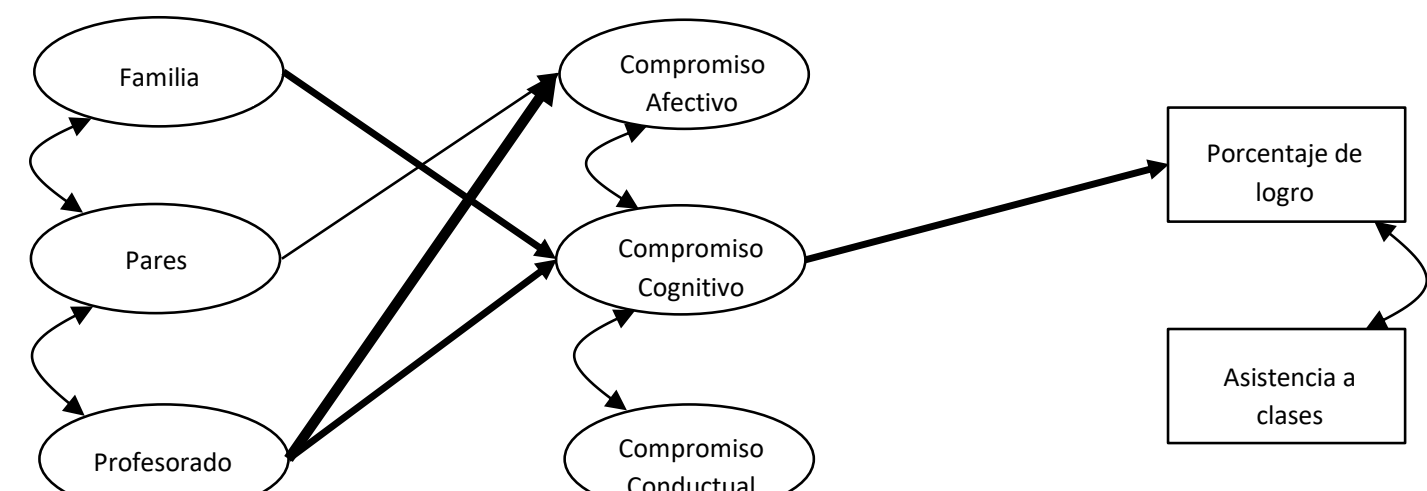

Factores Contextuales

Compromiso Escolar

Rendimiento escolar

Figura 5. Diagrama de rutas estadísticamente significativas del Modelo teórico de mediación del Compromiso Escolar en la relación entre Factores Contextuales y Rendimiento Escolar, en participantes de Perú

\footnotetext{
Efecto de magnitud alta $(\geq .50)$

Efecto de magnitud media $(>.30 \mathrm{y}<.50)$

Efecto de magnitud baja $(\leq .30)$
}

sobre el compromiso escolar cognitivo $(\gamma=.600$, $p<.001)$, y tres efectos de magnitud media, el efecto del factor contextual profesorado sobre el compromiso escolar afectivo $(\gamma=.498, p<.001)$, el efecto del compromiso escolar cognitivo sobre la asistencia a clases $(\beta=.355, p<.001)$ y el efecto del factor contextual pares sobre el compromiso escolar afectivo $(\gamma=.301, p<.001)$.

En la muestra de participantes de España, el modelo (Figura 4) presentó un efecto de magnitud alta entre el factor contextual profesorado sobre el compromiso escolar afectivo $(\gamma=.513, p<.001)$. Además, se evidencian tres efectos de magnitud media, el efecto del factor contextual pares sobre el compromiso escolar afectivo $(\gamma=.418, p<.001)$, el efecto del factor contextual profesorado sobre el compromiso escolar cognitivo $(\gamma=.410, p<.001)$ y el efecto del factor contextual familia sobre el compromiso escolar conductual $(\gamma=.395, p<.001)$.

En la muestra de participantes de Perú, el modelo (Figura 5) presentó un efecto de magnitud alta entre el Factor contextual profesorado sobre el compromiso escolar afectivo $(\gamma=.705, p<.001)$. Además, se evidencian cuatro efectos de magnitud media, el efecto del factor contextual profesorado sobre el compromiso escolar conductual $(\gamma=.465$, $p<.001$ ), el efecto del factor contextual familia sobre el compromiso escolar cognitivo $(\gamma=.390$, $p<.001)$, el efecto del compromiso escolar cognitivo sobre el porcentaje de logro $(\beta=.383$, 


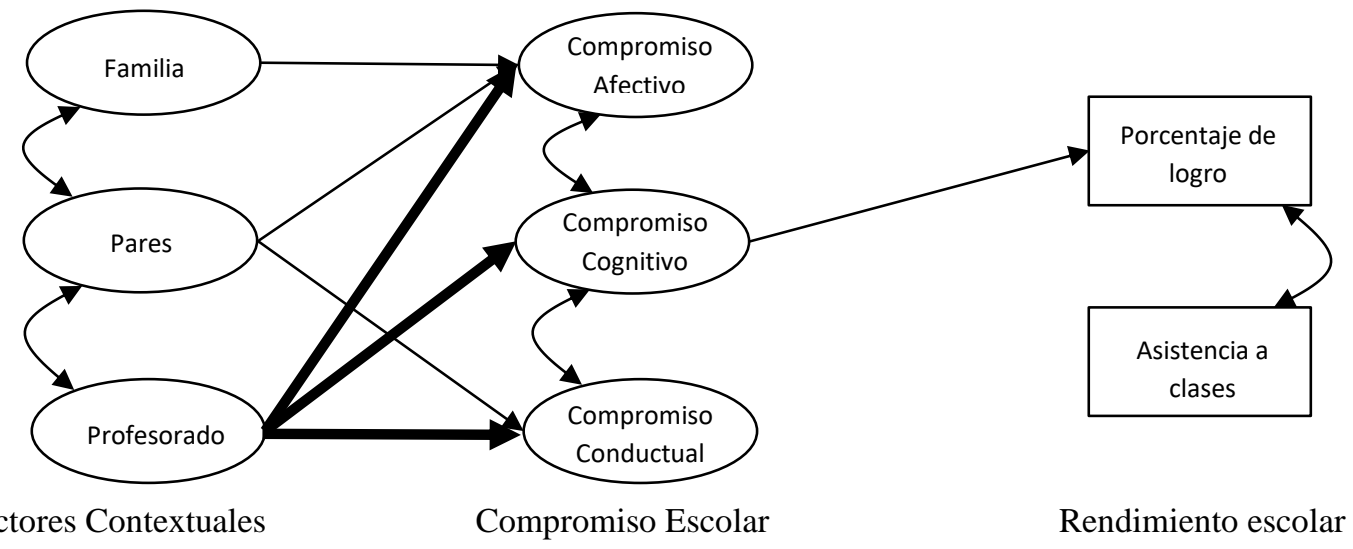

Figura 6. Diagrama de rutas estadísticamente significativas del Modelo teórico de mediación del Compromiso Escolar en la relación entre Factores Contextuales y Rendimiento Escolar, en participantes de Uruguay

Efecto de magnitud alta $(\geq .50)$

- Efecto de magnitud media $(>.30$ y $<.50)$

— Efecto de magnitud baja $(\leq .30)$

Tabla 6. Efectos estadísticamente significativos del modelo en los 5 países.

\begin{tabular}{|c|c|c|c|c|c|}
\hline Efecto & Chile & Colombia & España & Perú & Uruguay \\
\hline Familia $\rightarrow$ Afectivo & ns & ns & ns & ns & 0.153 \\
\hline Familia $\rightarrow$ Cognitivo & 0.248 & 0.190 & 0.211 & .390 & ns \\
\hline Familia $\rightarrow$ Conductual & ns & 0.289 & $\underline{0.395}$ & $\overline{\mathrm{ns}}$ & ns \\
\hline Profesorado $\rightarrow$ Afectivo & $\underline{0.669}$ & $\underline{0.498}$ & $\overline{0.513}$ & $\underline{0.705}$ & $\underline{0.611}$ \\
\hline Profesorado $\rightarrow$ Cognitivo & $\overline{0.645}$ & $\overline{0.600}$ & $\overline{0.410}$ & $\overline{0.358}$ & $\overline{0.666}$ \\
\hline Profesorado $\rightarrow$ Conductual & $\overline{0.459}$ & $\overline{0.255}$ & $\overline{0.243}$ & $\underline{0.465}$ & $\overline{0.938}$ \\
\hline Pares $\rightarrow$ Afectivo & $\overline{0.203}$ & $\underline{0.301}$ & $\underline{0.418}$ & $\overline{0.243}$ & $\overline{0.241}$ \\
\hline Pares $\rightarrow$ Cognitivo & -0.179 & ns & ns & ns & ns \\
\hline Pares $\rightarrow$ Conductual & ns & -0.172 & ns & ns & -0.270 \\
\hline Afectivo $\rightarrow$ Logro & -0.132 & ns & ns & ns & $\mathrm{ns}$ \\
\hline Afectivo $\rightarrow$ Asistencia & 0.148 & $\mathrm{~ns}$ & ns & $\mathrm{ns}$ & ns \\
\hline Cognitivo $\rightarrow$ Logro & $\underline{0.523}$ & 0.210 & $\mathrm{~ns}$ & $\underline{0.383}$ & 0.279 \\
\hline Cognitivo $\rightarrow$ Asistencia & ns & $\underline{0.355}$ & ns & ns & ns \\
\hline Conductual $\rightarrow$ Logro & ns & $\overline{0.277}$ & 0.144 & ns & ns \\
\hline Conductual $\rightarrow$ Asistencia & 0.267 & ns & ns & ns & ns \\
\hline
\end{tabular}

\section{Negritas y subrayado $=$ Efecto de magnitud alta.}

$\underline{\text { Subrayado }=E f e c t o ~ d e ~ m a g n i t u d ~ m e d i a . ~}$

ns=Efecto estadísticamente no significativo.

$p<.001)$ y el efecto del factor contextual profesorado sobre el compromiso escolar cognitivo $(\gamma=.358, p<.001)$.

En la muestra de participantes de Uruguay, el modelo (Figura 6) presentó tres efectos de magnitud alta, el efecto del factor contextual profesorado sobre el compromiso escolar conductual $(\gamma=.938, p<.001)$, sobre el compromiso escolar cognitivo $(\gamma=.666, p<.001)$ y sobre el compromiso escolar afectivo $(\gamma=.611, p<.001)$.

En la Tabla 6 se presenta un resumen de los efectos estadísticamente significativos en cada país.

\section{Discusión}

El modelo teórico de mediación del compromiso escolar en la relación entre factores contextuales y rendimiento escolar presenta un ajuste aceptable en los cinco países. Siguiendo lo señalado por Ondé Pérez (2019), es importante tener en consideración que el modelo propuesto es un modelo posible de causalidad de variables sobre el rendimiento escolar, pero en ningún caso el único. Sin embargo, nos permite tener una aproximación empírica de algunas variables que afectan el rendimiento escolar, que es consistente con la teoría y estudios previos (Miranda-Zapata et al., 2018).

Entre los resultados destaca que en los cinco países, el factor contextual del profesorado presenta efectos estadísticamente significativos hacia las tres dimensiones del compromiso escolar, variando en su magnitud. La importancia de la relación entre estudiantado y profesorado en cuando al compromiso escolar es un aspecto clave que ha sido resaltado en la literatura científica. 
Por ejemplo, Havik y Westergård (2020) reportan que el apoyo emocional de los profesores fue el aspecto que mostró la asociación más fuerte con el compromiso escolar de los estudiantes que participaron en el estudio. De forma general, es un resultado consistente a lo largo de la literatura disponible (Martin \& Collie, 2019), y queda claramente establecida en la revisión sistemática realizada por Quin, donde reportan como una mejor relación entre estudiantes y profesorado estuvo asociada con un mayor compromiso escolar.

Se comprueba que en los cinco países el compromiso escolar se ve afectado por factores de contexto y al menos una dimensión del compromiso escolar tiene efectos sobre el porcentaje de logro como manifestación de rendimiento escolar. Siendo el CE, por lo tanto, una variable relevante de estudiar y buscar intervenir para favorecer su fortalecimiento en los estudiantes. De este modo, los resultados encontrados son consistentes con lo reportado en la literatura científica al respecto (Gutiérrez, Sancho, Galiana, \& Tomás, 2018; Korobova \& Starobin, 2015; Li, Chen \& Li, 2020; MirandaZapata et al., 2018). Esta situación queda claramente establecida en los resultados del metaanálisis realizado por Lei et al. (2018) con 69 estudios independientes, donde concluyen que la relación entre el compromiso escolar y el logro académico es moderadamente fuerte, y esta relación se encuentra al diferenciar entre componentes del compromiso escolar en casi todos los casos.

Por su parte, la asistencia a clases no recibe efecto del compromiso escolar en al menos tres de los países incluidos en este estudio, lo cual no pareciera tener mayor relevancia si se toma en consideración que la situación mundial actual en reacción a la pandemia por COVID-19 hará de la asistencia un aspecto que cambiará su valor, debido a la necesidad de cuidado y protección del estudiantado. Lo que podría llevar a valorar que un/a estudiante se quede en casa si presenta alguna molestia que pueda estar asociada a sintomatología por COVID-19.

Para futuros estudios, se considera conveniente incorporar muestras de mayor tamaño, que puedan tener características que permitan generalizar los resultados a cada contexto de los países incluidos en el estudio.

\section{Agradecimiento}

Agradecemos el trabajo en la recolección de datos por parte de José Alfredo Aparicio (Universidad del Norte, Colombia), Sergio Domínguez (Universidad San Martín de Porres, Perú), Fernando Acevedo (Universidad de la República, Uruguay) y Rosa María Díaz (Universidad Pablo de Olavide, España).

\section{Financiamiento}

Este artículo ha recibido el apoyo de los proyectos ANID/FONDECYT 1210172, FONDEF ID14I20078 y FONDEF IT 19I0012, financiado por la Comisión Nacional de Investigación Científica y Tecnológica CONICYT, Chile.

\section{Referencias}

Acevedo, F. (2020). Factores explicativos del abandono de los estudios en la educación superior en contextos socio-académicos desfavorables. Revista Española de Pedagogía, 78(276), 195-212. doi:10.22550/REP78-2-2020-02

Ansong, D., Okumu, M., Bowen, G. L., Walker, A. M., \& Eisensmith, S. R. (2017). The role of parent, classmate, and teacher support in student engagement: Evidence from Ghana. International Journal of Educational Development, 54, 51-58. doi:10.1016/j.ijedudev.2017.03.010

Appleton, J. J., Christenson, S. L., \& Furlong, M. J. (2008). Student engagement with school: Critical conceptual and methodological issues of the construct. Psychology in the Schools, 45(5), 369-386. doi:10.1002/pits.20303

Archambault, I., Janosz, M., Fallu, J., \& Pagani, L. (2009). Student engagement and its relationship with early high school dropout. Journal of Adolescence, 32(3), 651-670. doi:10.1016/j.adolescence.2008.06.007

Dotterer, A. M., McHale, S. M., \& Crouter, A. C. (2007). Implications of out-of-school activities and school engagement in African American adolescents. Journal of Youth and Adolescence, 36(4), 391-401. 
doi:10.1007/s10964-006-9161-3

Espinoza, O., González, L., McGinn, N., \& Castillo, D. (2020). Engaging dropouts with differentiated practices: Some evidence from Chile. Research Papers in Education. doi:10.1080/02671522.2020.1736615

Fernández-Zabala, A., Goñi, E., Camino, I., \& Zulaika, L. M. (2016). Family and schoolcontext in school engagement. European Journal of Education and Psychology, 9(2), 47-55. doi:10.1016/j.ejeps.2015.09.001

Flotts, M. P., Manzi, J., Jiménez, D., Abarzúa, A., Cayuman, C., \& García, M. J. (2016). Informe de Resultados TERCE, Logros de Aprendizaje: Laboratorio Latinoamericano de Evaluación de la Calidad de la Educación. Santiago Orealc/Unesco. Recuperado de http://unesdoc.unesco.org/images/0024/00243 5/243532S.pdf

Fredricks, J. A., Blumenfeld, P., \& Paris, A. (2004). School engagement: Potential of the concept, state of the evidence. Review of Educational Research, 74(1), 59-109. doi:10.3102/00346543074001059

Fredricks, J. A., Filsecker, M., \& Lawson, M. A. (2016). Student engagement, context, and adjustment: Addressing definitional, measurement, and methodological issues. Learning and Instruction, 43, 1-4. doi:10.1016/j.learninstruc.2016.02.002

Froiland, J. M., \& Worrell, F. C. (2016). Intrinsic motivation, learning goals, engagement, and achievement in a diverse high school. Psychology in the Schools, 53(3), 321-336. doi:10.1002/pits.21901

González, A., Paoloni, P., Donolo, D., \& Rinaudo, C. (2015). Behavioral engagement and disaffection in school activities: Exploring a model of motivational facilitators and performance outcomes. Anales de Psicología, 31(3), 869-878.

Gutiérrez, M., Sancho, P., Galiana, L., \& Tomás, J. M. (2018). Autonomy support, psychological needs satisfaction, school engagement and academic success: A mediation model. Universitas Psychologica, 17(5), 1-12. doi:10.11144/Javeriana.upsy17$5 . a s p n$
Havik, T., \& Westergård, E. (2020). Do Teachers Matter? Students' perceptions of classroom interactions and student engagement. Scandinavian Journal of Educational Research, 64(4), 488-507. doi:10.1080/00313831.2019.1577754

Kiefer, S. M., Alley, K. M., \& Ellerbrock, C. R. (2015). Teacher and peer support for young adolescents' motivation, engagement and school belonging. RMLE Online, 38(8), 1-18. doi:10.1080/19404476.2015.11641184

Kline, R. B. (2010). Principles and practice of structural equation modeling ( $3^{\text {rd }}$ ed.). New York: The Guilford Press.

Korobova, N., \& Starobin, S. S. (2015). A comparative study of student engagement, satisfaction, and academic success among international and American students. Journal of International Students, 5(1), 72-85. Recuperado de https://www.ojed.org/index.php/jis/article/vie $\mathrm{w} / 444$

Lam, S., Wong, B., Yang, H., \& Liu, Y. (2012). Understanding student engagement with a contextual model. En S. L. Christenson, A. L. Reschly, \& C. Wylie (Eds.), Handbook of research on student engagement (pp. 403420). New York, NY: Springer.

Lara, L., Saracostti, M., Navarro, J.-J., de-Toro, X., Miranda-Zapata, E., Trigger, J. M., \& Fuster, J. (2018). Compromiso escolar: Desarrollo y validación de un instrumento. Revista Mexicana de Psicología, 35(1), 52-62.

Lei, H, Cui., Y., \& Zhou, W. (2018). Relationships between student engagement and academic achievement: A meta-analysis. Social Behavior and Personality: An International Journal, 46(3), 517-528. doi:10.2224/sbp.7054

$\mathrm{Li}, \mathrm{Ch} . \mathrm{H}$. (2014). The performance of MLR, USLMV, and WLSMV estimation in structural regression models with ordinal variables (PhD Thesis). Michigan State University: Michigan, United States.

Li, L., Chen, X., \& Li, H. (2020). Bullying victimization, school belonging, academic engagement and achievement in adolescents in rural China: A serial mediation model. Children and Youth Services Review, 113. doi:10.1016/j.childyouth.2020.104946 
Marsh, H. W., Hau, K. T., \& Grayson, D. (2005). Goodness of fit in structural equation models. En A. Maydeu-Olivares, \& J. J. McArdle (Eds.), Multivariate applications book series. Contemporary psychometrics: A Festschrift for Roderick P. McDonald (pp. 275-340). Lawrence Erlbaum Associates Publishers.

Martin, A. J., \& Collie, R. J. (2019). Teacherstudent relationships and students' engagement in high school: Does the number of negative and positive relationships with teachers matter? Journal of Educational Psychology, 111(5), 861-876.

doi: $10.1037 /$ edu0000317

Martínez-Novillo, J. (2017). La construcción del «fracaso escolar» en España. Génesis y cristalización de un problema social. Revista de Sociología, 102(3), 477-507.

doi: $10.5565 / \mathrm{rev} /$ papers. 2297

Ministerio de Educación y Consejo Nacional de Educación (2007). Proyecto Educativo Nacional al 2021: La educación que queremos para el Perú. Lima: Consejo Nacional de Educación. Recuperado de http://www.minedu.gob.pe/DeInteres/xtras/PE N-2021.pdf

Miranda-Zapata, E., Lara, L., Navarro, J.-J., Saracostti, M., \& de-Toro, X. (2018). Modelización del efecto del compromiso escolar sobre la asistencia a clases y el rendimiento escolar. Revista de Psicodidáctica, 23(2), 102-109. doi:10.1016/j.psicod.2018.02.003

Ondé, D. (2019). Revisión del Concepto de Causalidad en el Marco del Análisis Factorial Confirmatorio. Revista Iberoamericana de Diagnóstico y Evaluación - e Avaliação Psicológica, 54(1), 103-118.

Quin, D. (2017). Longitudinal and contextual associations between teacher-student relationships and student engagement: A systematic review. Review of Educational Research, 87(2), 345-387. doi:10.3102/0034654316669434

Quin, D., Heerde, J. A., \& Toumbourou, J. W. (2018). Teacher support within an ecological model of adolescent development: Predictors of school engagement. Journal of School Psychology, 69, 1-15. doi:10.1016/j.jsp.2018.04.003
Roundfield, K. D., Sánchez, B., \& McMahon, S. D. (2016). An ecological analysis of school engagement among urban, low-income latino adolescents. Youth and Society, 50(7). doi:10.1177/0044118X16639986

Reschly, A. L., \& Christenson, S. L. (2012). Jingle, jangle and conceptual haziness: Evolution and future directions of the engagement construct. En S. L. Christenson, A. L. Reschly, \& C. Wylie (Eds.), Handbook of research on student engagement (pp.3-20). New York: Springer.

Rial, A., Varela, J., Abalo, J., \& Lévy, J. P. (2006). El análisis factorial confirmatorio. In J. P. Lévy, \& J. Varela (Coords.), Modelización con Estructuras de Covarianzas en Ciencias Sociales: Temas esenciales, avanzados y aportaciones especiales (pp. 1130). A Coruña: Netbiblo.

Sahin, S., Arseven, Z., \& Kiliç, A. (2016). Causes of Student Absenteeism and School Dropouts. International Journal of Instruction, 9(1), 195-210. Recuperado de http://www.eric.ed.gov/contentdelivery/servle t/ERICServlet?accno=EJ1086967

Salanova, M., Schaufeli, W., Martínez, I., \& Bresó, E. (2010). How obstacles and facilitators predict academic performance: The mediating role of study burnout and engagement. Anxiety, Stress \& Coping, 23(1), 53-70. Recuperado de http://www.want.uji.es/wpcontent/uploads/2017/03/2010_SalanovaSchaufeli-Mart\%C3\%AdnezBres\%C3\%B3.pdf

Tomás, J. M., Gutiérrez, M., \& Fernández, I. (2016). Predicción de la satisfacción y el rendimiento escolar: El compromiso como mediador. Revista Búsqueda, 3(16), 7-19.

Veiga, F. H., Robu, V., Conboy, J., Ortiz, A., Carvalho, C., \& Galvão, D. (2016). Students' engagement in school and family variables: A literature review. Estudios de Psicología (Campinas), 33(2), 187-197. doi:10.1590/1982-02752016000200002

Weiss, C. C., Carolan, B. V., \& Baker-Smith, E. C. (2010). Big school, small school: (Re)Testing assumptions about high school size, school engagement and mathematics 
achievement. Journal of Youth Adolescence, 39(2), 163-176.

doi:10.1007/s10964-009-9402-3

Yusof, N., Ang, R. P., \& Oei, T. P. S. (2017). The psychometric properties of the school engagement measure in adolescents in Singapore. Journal of Psychoeducational Assessment, 35(5).

doi: $10.1177 / 0734282916639441$ 